\title{
La imaginación narrativa de Martha Nussbaum ante la crítica académica
}

\author{
Martha Nussbaum's concept of narrative imagination facing \\ academic criticism
}

Luis Alfonso Argüello Guzmán plotino2080@hotmail.com

http://orcid.org/000-0002-8799-4102

Instituto de Educación a Distancia; Universidad del Tolima (Colombia)

\section{Resumen}

Este artículo presenta una revisión documental y bibliográfica sobre autores que han elaborado puntos a favor y en contra sobre la categoría de imaginación narrativa de Martha Craven Nussbaum. Esta revisión se desarrolla en tres secciones: la primera presenta los rasgos generales de los planteamientos de Martha Nussbaum sobre la Imaginación Narrativa. La segunda es una revisión descriptiva de los planteamientos críticos asociados a la categoría de Nussbaum, con dos debates críticos: de la ética crítica y de la literatura como filosofía moral. La tercera parte de este artículo centra su interés general en las posibilidades conceptuales de la imaginación narrativa y, de manera particular, en el potencial del encuentro de ámbitos de lectura en la educación superior. 
Palabras clave: Martha Nussbaum; imaginación narrativa; crítica académica; ámbitos de lectura.

\section{Abstract}

This article presents a bibliographic revision about some authors who have presented some claims in favor and against Martha Nussbaum's concept of narrative imagination. The present revision is developed in three stages: the first one presents the general features of Martha Nussbaum's claims on the concept of Narrative Imagination. The second stage corresponds to a descriptive revision of the critical statements associated to Nussbaum's category in question, which are mainly presented in two critical issues: the one from critical ethics and the other comes from literature as moral philosophy. The third part of this article centers its general interest on the conceptual possibilities of narrative imagination and particularly on the potential it has in the encounter of reading spheres in higher education

Keywords: Martha Nussbaum; narrative imagination; academic criticism; reading spheres.

Este artículo es una revisión documental y bibliográfica sobre autores que han estudiado la categoría de imaginación literaria de Martha C. Nussbaum. Corresponde a un avance de la tesis doctoral titulada La comprensión lectora narrativa en la formación de licenciados en Lengua castellana, para optar al título de Doctor en Humanidades, Humanismo y Persona (énfasis en Antropología Pedagógica), de la Universidad San Buenaventura, sede Bogotá. El desarrollo de la indagación documental y bibliográfica se divide en tres secciones: la primera comprende la revisión descriptiva del estado del arte; la segunda comprende dos debates asociados a la categoría de Nussbaum: el de la ética crítica y el de la Literatura como filosofía moral; la tercera se refiere a las posibilidades teóricas de la categoría.

La revisión de fuentes textuales está sujeta a dos niveles: documentales y bibliográficas. Las fuentes documentales son del tipo tesis de maestría y tesis de doctorado; las fuentes bibliográficas se seleccionan a partir de artículos de publicaciones seriadas, libros y capítulos de libro que contienen análisis sobre la categoría. Por último, a partir de la paráfrasis descriptiva y los debates teóricos, se presentan las posibilidades teóricas asociadas a la Imaginación narrativa que se focalizan en la importancia de la lectura literaria en educación superior. 
Unos de los aspectos que subyacen en el problema de indagación, es mostrar como en la categoría de imaginación narrativa se encuentra en potencia la posibilidad de construir un modelo de lectura literaria que considera al lector en toda su integridad personal, justo en el momento de la comprensión lectora de obras literarias narrativas. Esta posibilidad de un modelo tiene sus seguidores desde la perspectiva de lo que puede aportar la filosofía moral a la lectura misma, no obstante que los críticos atacan la extrema cercanía y falta de delimitación entre los análisis literarios y la filosofía moral. El propósito central de este artículo es revisar a la categoría en mención y revisar las aproximaciones críticas que han surgido desde su planteamiento, hasta definir las tendencias que subyacen en su planteamiento y las posibilidades creativas, interpretativas y comunicativas de la categoría de imaginación narrativa.

\section{La imaginación narrativa en Martha Nussbaum}

Para Martha Nussbaum (1997) la imaginación literaria cumple un rol ético en el plano de su ejemplificación en la deliberación pública, tomando como criterio de selección de análisis la selección de obras literarias narrativas, a partir de las cuales construye un modelo de lectura que empareja el análisis literario con el análisis moral. Lo que está en juego al plantearse la imaginación literaria en el horizonte de la deliberación pública, es resaltar su poder narrativo, desde este tipo de análisis.

Creer que la imaginación literaria tiene un poder para el desarrollo de las Humanidades, no significa que al realizar los análisis literarios con intención filosófica, deban eliminarse las particularidades de la obra literaria, ni erradicar el análisis de la filosofía moral (Diamond, 1983) del campo de lo literario. Tanto en la lectura literaria como en el análisis de la filosofía moral, se mantiene una relación de proximidad que facilita la lectura y a la vez guía al lector al sentido o los sentidos de las obras literarias narrativas

Lo común en la anterior relación de proximidad es que la lectura literaria de obras narrativa permite confrontar la vida emocional de cada lector al momento de enfrentarse con el mundo de ficción de la obra. Es en la obra literaria narrativa, con su poder narrativo, donde podría potenciarse su valor como un despliegue de capacidades, por lo que es en las novelas (Johnson, 2004) donde Nussbaum intenta encontrar objetos de análisis para la deliberación pública. La lectura de obras narrativas literarias, desde la imaginación narrativa produce un encuentro de ámbitos de lectura: desde el ámbito del mundo de ficción narrativa y desde el mundo del lector. 
Para lograr lo anterior, Nussbaum escribe que se requieren tres capacidades para el cultivo de la humanidad: la primera concierne al examen de uno mismo y de las propias tradiciones, la segunda a cómo verse a uno mismo no sólo en relación con un grupo o tradición propia sino como vinculado con otros grupos y

\begin{abstract}
La tercera destreza que debe poseer el ciudadano, estrechamente relacionada con las dos primeras, se puede llamar imaginación narrativa. Esto significa la capacidad de pensar cómo sería estar en el lugar de otra persona, ser un lector inteligente de la historia de esa persona, y comprender las emociones, deseos y anhelos que alguien así pudiera experimentar (Nussbaum, 2005: 30).
\end{abstract}

En esta definición se pueden inferir tres aspectos fundamentales sobre la imaginación narrativa: tiene como punto de origen obras en forma de relatos, trata de cómo un lector lee esos relatos y aborda el asunto de la imaginación como capacidad para comprender significados y emociones. En consecuencia, una primera aproximación a la imaginación literaria se relaciona con la capacidad del lector para reconocer la vida de otras personas a través de obras narrativas.

Con este reconocimiento surge la empatía como probabilidad que destaca el papel de la literatura en la consecución de esta comprensión lectora que al mismo tiempo es reconocimiento: "si la literatura es una representación de las posibilidades humanas, las obras de la literatura que escogemos inevitablemente responderán a nuestro sentido de quiénes somos y quiénes podríamos ser, y lo que desarrollarán más aún" (Nussbaum, 2005: 142). Por lo tanto, el papel de la literatura expresada en narrativa literaria, cobra importancia en la medida en que se evidencia la vida, pensamientos y emociones de otras personas en forma de relatos.

La comprensión lectora de estos relatos literarios es lo que hace de la imaginación literaria un eslabón entre la comprensión del lector y la comprensión de la novela. Por tal razón, un segunda definición posible de imaginación narrativa se relaciona con la capacidad del lector para comprender las formas de pensar y sentir de otras personas a través de obras narrativas literarias, ante lo cual "La gran contribución que tiene que hacer la literatura a la vida del ciudadano es su capacidad de arrancar de nuestras obtusas imaginaciones un reconocimiento de aquellos que no son nosotros, tanto en circunstancias concretas como en la manera de pensar y sentir (Nussbaum, 2005: 148).

En estas circunstancias, comprensión lectora de obras narrativas es a su vez una acción de reconocimiento, y hace de la imaginación literaria un modo de comprensión asociada a la 
experiencia literaria del lector y a la experiencia ficcional configurada en la novela. Este modo de comprensión permite la preparación para la interacción entre el mundo ficcional de la novela y la experiencia del lector: así la comprensión lectora se constituye en el punto de arranque para la transformación de la imaginación narrativa como un encuentro de ámbitos de lectura.

Por lo que sea presentado hasta el momento, es en el momento del acercamiento entre la acción comprensiva que surge de la experiencia del lector y la realidad ficcional comprendida de la novela donde se produce el encuentro de ámbitos de lectura y se crean las condiciones para lograr que la imaginación literaria sea una capacidad humana asociada a la lectura, a la vez que se convierte en el encuentro relacional que facilita la formación de un lector que cumple sus obligaciones como persona y como ciudadano.

\section{La imaginación literaria ante la crítica académica}

\section{Paráfrasis textual del corpus crítico}

Voice (1994) se opone al reclamo de Nussbaum sobre que algunas obras literarias son también obras de filosofía moral. Esta oposición se ancla en el argumento de que el reclamo falla al momento de reconocer el ámbito concreto de la filosofía moral, sus preocupaciones, sus tareas y sus métodos de investigación. En este sentido, la teoría moral y las características de los textos literarios expresan sus verdades con un alcance mucho más estrecho del que Nussbaum reconoce. Su crítica radica en cómo se intenta suplir el análisis filosófico mismo acudiendo a obras literarias, con lo cual se delimita la lectura literaria a un carácter ejemplificante.

Diamond (1999) continúa abordando los reclamos de Nussbaum, en primer lugar sobre cómo vivir y a la vez cómo su transmisión textual en la novelas depende de la estructura formal de ese texto; esto significa que si acepta este argumento, la visión de la vida encaja en cierto de textos y no otros. En segundo lugar, sobre el hecho de hay ciertas visiones morales que sólo pueden ser expresadas por las novelas, por lo cual la narrativa de las novelas son parte del corpus de análisis de la filosofía moral. Tanto para Voice y como para Diamond, el núcleo de sus críticas a Nussbaum radica en la manera como restringe el análisis moral a la ejemplificación de la vida de un personajes de novela.

Von Wright (2002) apuntala que la imaginación narrativa no sólo requiere conocimiento y razonamiento como lectores de un texto narrativo, sino amor y compasión para su lectura. En ese plan, la imaginación narrativa es una herramienta de lectura para el entendimiento de lo humano. Ese tipo de acercamiento a la lectura podría promover el examen crítico y la reflexión, 
lo cual sería útil para comprender la vida que vivimos en orden a cultivar lo que nos toca y nos afecta como lectores. De nuevo, la vida representada en la vida de unos personajes de novela. Horton (2003) recapitula sobre este punto, con el argumento que desde el punto de vista de los novelistas, la abstracción filosófica es una intrusa en el imperativo estético de la imaginación narrativa y desde la perspectiva de los filósofos, las novelas son teorizaciones innecesarias que no contribuyen con los análisis filosóficos. En consecuencia, indagar por la imaginación literaria es tanto análisis literario como discernimiento ético

Hamilton (2004) argumenta sobre el valor del discernimiento ético en el análisis literario y sostiene que la literatura promueve clases de juicio específico, respeto del valor cognitivo de las emociones y de la empatía, ya que son fundamentales para la clase de juicio ético que Nussbaum postula. Por consiguiente, aclarar que las novelas de Henry James muestran estos mismos valores éticos lleva a identificarlas por su utilidad para la instrucción ética, lo cual hace de la imaginación un potencial de discernimiento ético. Singleton (2006) se concentra en la conexión aliada entre las novelas de James y la ética de Aristóteles, de manera particular sobre cuatro atributos que sirven de encuentro entre el novelista y el filósofo: ejemplo de buena elección en las obras, ilustración de la dirección de pensamiento para el discernimiento de lo particular, desarrollo de la imaginación y fuente para el entendimiento de las emociones Antaki (2012), en esta línea, describe cuatro formas de imaginación que son vitales para la comprensión filosófica y literaria: teórica (imaginación como síntesis), progresiva (imaginación como empatía), transformativa (imaginación como invención) y nostálgica (imaginación como armonía). Es en la segunda forma, la imaginación como sintonía, donde ubica la obra de Martha Nussbaum referida a la imaginación literaria y anota que en Justicia Poética (1997), la imaginación tiende a lo moral y a la justicia social, enfatizando que este modo de leer tiende al instrumentalismo de la novela para el entendimiento de la empatía de los lectores. Otra vez, la crítica recae sobre el hecho de comparar la vida novela de unos personajes como modelo de la vida fáctica misma: esa es su crítica al instrumentalismo moral de la lectura de novelas.

Mcgregor (2013) expone dos objeciones particulares de la autonomía de la literatura: que el valor moral es parte del valor literario de las novelas realistas y que la lectura de estas obras literarias tiene el significado de mejora moral, con lo cual se evidencia mostrando que Nussbaum se equivoca al admitir la relación directa entre valor moral y valor literario. En el mismo horizonte de objeciones, Robbins (2015) examina el argumento de la profesora Nussbaum(1995), sobre el papel positivo del compromiso literario en el proceso de formación del juicio moral y político, sobre la base de que la experiencia de lecturas de novelas ofrece la oportunidad de comprometer nuestra empatía con un beneficio moral. Por el contario, contrario, tomando como ejemplo la ciencia ficción y en particular las novelas de Úrsula K. Le Guin, 
expone que los pretendidos beneficios de la lectura juiciosa son incompatibles con los requerimientos de este tipo de novelas. En este mismo sentido, Bevan (2006) desarrolla la idea del valor de la literatura como un ejemplo del poder de la imaginación en los procesos educativos, haciendo de la literatura un valor potencial que es aplicable al currículo sensible, retomando para su análisis la obra de Maxime Geene, Wayne Booth Martha Nussbaum.

Hidalgo (2010) analiza la imaginación literaria como proyección en el otro, con el planteamiento de preguntas sobre las implicaciones éticas de la literatura, sobre la mirada humanista de los textos literarios, ejemplificando el asunto en la obra Las estrellas son negras del escritor Arnoldo Palacios, para producir un acercamiento al discurso acerca del significado de la literatura. Ahí radica, según Hidalgo, el potencial de la imaginación literaria para la educación literaria. En este punto, Lourens (2009) se detiene en el argumento de Nussbaum sobre cómo comunicar la naturaleza de la existencia ética: se centra en el propósito de combinar literatura de ficción con filosofía moral, para lo cual se focaliza en tres aspectos: los valores humanos son plurales e inconmensurables; las situaciones particulares cumplen un papel importante en la deliberación ética; las emociones y la imaginación forman parte de nuestra existencia ética. En esta misma línea de exposición, Fonnegra (2012) desarrolla la idea de que desde la sabiduría práctica aristotélica no hay antagonismo entre texto filosófico y texto literario dando cabida a la narrativa literaria. Esta aceptación permite que en la obra literaria, mediante las emociones y la facultad imaginativa, se amplíe la experiencia humana.

Hänes y Jansen (2001), continúan con este desarrollo expositivo sobre la relación entre Literatura y Filosofía, para esbozar dos ideas: que la literatura que leemos forma nuestras creencias morales y que el reclamo de Nussbaum de la literatura en la teoría de lo "bueno", debe complementarse con una lista de actividades, capacidades y contrastes de la vida humana, para de esta forma delimitar el papel de la literatura y la filosofía en el análisis de lo bueno. Maxwell (2006), en el marco de lo "bueno", recoge el argumento de Nussbaum de restaurar a la lectura de novelas en correspondencia con la aproximación a los lazos de compasión basados en la solidaridad entre ciudadanos y dice que el valor educacional del estudio de la novela es necesario cuando fallan otras formas de apreciación imaginativa y por lo tanto, es una forma entre otras, de aproximación a la compasión.

Clavel (2012) interpone dos objeciones al valor de las novelas para el discernimiento ético: la primera apunta a delimitar cuáles textos merecen entrar al corpus del análisis filosófico; la segunda a la articulación entre literatura y filosofía sobre la unidad entre estilo del argumento y su contenido, sobre el rol de las emociones y sobre las situaciones particulares. Bermúdez (2014) sostiene la relación entre literatura y la formación de ciudadanos, al tomar como parámetro de análisis los planteamientos de Martha Nussbaum y Helen Modzelewski sobre la 
imaginación narrativa como parte de los procesos de formación en la educación ciudadana, a través de la empatía y las emociones.

Koopman y Hakemulder (2015) esbozan un modelo de lectura literaria que surge de la idea de lectura como una forma de asumir la empatía y de la idea de desfamiliarización de las características narrativas de un texto. Lo que está en juego en este tipo de análisis críticos sobre la categoría de imaginación narrativa en Nussbaum, es evaluar las nociones de empatía y auto-reflexión (conceptos desarrollados por Martha Nussbaum, aunque los autores no lo mencionan como punto de partida) a partir de la lectura.

\section{Debates}

\section{El debate de la ética crítica}

El debate de la ética crítica gira en torno a dos asuntos: sobre si es posible juzgar una obra literaria con argumentos de la razón práctica y si leer novelas forma mejores ciudadanos en una democracia (Stow, 2000). Este debate tuvo como opositores en un lado a Richard Posner (1997, 1998), que cuestionó la legitimidad de la ética crítica, y Martha Nussbaum (1998) con Wayne Booth (1998) en el otro, quienes defendieron el valor la ética crítica para los análisis en la relación literatura y filosofía moral.

Para Posner existen dos objeciones a la intención de que la literatura sea parte de estos análisis: la primera está referida a que la literatura no es exclusiva fuente de entendimiento moral y la segunda que no existen pruebas sobre el avance de la recepción en la deliberación moral al plantearse la relación entre literatura y filosofía moral. Lo que está en entredicho en esta discusión es la pertinencia académica de la Literatura y su contribución al análisis ético.

Las objeciones de Posner en este debate se enmarcan en lo que Carroll denominó trivialidad cognitiva (2000: 353-355), que se compone de dos dimensiones: la primera sobre la naturaleza de los argumentos morales y la segunda sobre que las obras literarias o de arte no implican mayor conocimiento en la filosofía moral. Aquí están enfrentadas las posturas de la ética de la justicia en Posner (centrada en la autonomía estética de la obra) contra la visión amplia de Nussbaum soportada en la ética aristotélica.

En este debate se definió el terreno de lo que Buell (1999) denominó el auge de la ética crítica y su relación con la literatura y la filosofía moral en el marco del estudio del valor moral de los textos literarios, la historia del pensamiento moral y la ética narrativa 


\section{El debate de la literatura como filosofía moral}

Para Nussbaum (1983) Henry James se transforma en el caso particular que sirve de eslabón entre la narrativa, de manera puntual las novelas, y la filosofía moral, con la reducción íntima a la deliberación moral. En este debate, del cual participan la misma autora, Wollheim (1983) y Putnam (1983), se puede extraer que lo que estuvo en cuestión era por un lado, el poder de la narrativa para logar que las novelas fueran esenciales en la filosofía moral y que las obras de ficción fueran fundamentales para la formación de la conciencia moral.

El primer punto estuvo liderado en el debate por Wollheim (1983) para quien la novela Golden Bowl de Henry James podía brindar la oportunidad de comprender si la literatura podía ser esencial en la filosofía moral a la vez que era filosofía moral. El argumento contrario, puso en cuestión que se recurriera a la lectura de novelas para potenciar la deliberación moral: para superar esta visión estrecha se necesitaba trascender de la idea de la historia de la novela como asunto moral a una visión de la novela como narrativa; de igual forma, se era necesario reconocer qué era moral y que no lo era. En este mismo camino, Putnam (1983) sostuvo que Nussbaum estaba en su derecho de ver las obras de ficción como formadoras de conciencia moral, no obstante que interpuso objeciones a la idea de oponer la ética aristotélica a la ética kantiana. Además, escribió, no era posible que una novela soportara el planteamiento de moralidad explícita, evidenciada en su historia: una obra de ficción, para Putnam, no podía ser confundida con un comentario de filosofía moral.

\section{Tendencias de la crítica a la imaginación narrativa}

Desde la obra crítica sobre la imaginación narrativa se puede extraer que existen cuestionamientos a la relación entre Filosofía y Literatura, de manera particular sobre cuáles son los criterios de selección de las obras que permiten desarrollar análisis morales: en la preferencia de Nussbaum por los textos narrativos, sobre por qué los textos literarios tendrían mayor influencia en el análisis ético que los mismo textos éticos. En complemento, se pone en cuestión el intento de borrar las fronteras entre filosofía y literatura, con mayor precisión, a los límites visibles entre los alcances del análisis literario y el involucramiento del análisis del discernimiento moral, con la transformación de la lectura literaria de las novelas realistas, en mecanismos de ejemplificación de los análisis de la filosofía moral.

De igual manera, se cuestiona que las novelas realistas sean una única fuente para la formación del juicio político y moral de los lectores, con lo cual se pretende lograr la empatía con la vida de otras personas a través de los personajes novelados, siempre desde en la 
experiencia lectora; en la medida que se produce un acercamiento entre lector y personajes, por cuanto la comprensión de otras vidas se desarrolla en el terreno de la ficción, las emociones y la empatía de vuelven parte integral del proceso de lectura literaria.

Por último, la crítica puntualiza que la imaginación narrativa en Martha Nussbaum tiende a lo moral y a la justicia social, por lo que el modo de lector que surge de la imaginación narrativa instrumentaliza la novela con fines de análisis moral. Esta instrumentalización descarga su fundamento analítico en las novelas de Henry James o Charles Dickens.

\section{Posibilidades de la imaginación narrativa}

La categoría de imaginación literaria contiene el potencial de un encuentro de ámbitos, el sentido de la ficción narrativa y el de la experiencia del lector, con lo cual se puede potenciar como un espacio de participación de los procesos de compresión lectora. Este espacio de participación crea las condiciones para hacer de la lectura un encuentro para compartir experiencia de lectura, a la vez que organiza en el lector la manera de aproximarse a la comprensión misma y a cómo enseñarla, como acontecimiento estético y literario.

Con la reducción de la acción educativa a experiencia estética, el encuentro de ámbitos de lectura de una obra literaria narrativa convierte la creación, la recepción y la comunicación de lo leído en juego imaginativo, que facilita al lector la comprensión del mundo, del mundo del texto y del mundo del lector mismo:

saber que la imaginación nos permite ser otros y ser nosotros mismos, descubrir que podemos pensarnos, nombrarnos, soñarnos, encontrarnos, conmovernos y descifrarnos en ese gran texto escrito a tantas voces por infinidad de autores a lo largo de la historia, es el que le otorga sentido a la experiencia literaria como expresión de nuestra común humanidad (Reyes, 2007: 13-14).

La comprensión lectora del sentido o los sentidos del texto literario es lo que posibilita la experiencia literaria del lector y transforma la imaginación narrativa en capacidad humana de imaginación lectora, a la vez que permite el encuentro entre los sentidos de la obra de la ficción y la experiencia del lector, con la mediación de la acción educativa como acción social que pone en juego la experiencia estética como creación, recepción y comunicación. Este carácter mediador de la experiencia estética y literaria, permite la participación en la actividad educativa como acción social creativa, receptiva y comunicativa: 
ni la creación (acción social), ni la recepción (acción moral) pueden separarse de la acción educativa (acción comunicativa) el sentido de la educación, el encuentro educativo, debe ser construido por ambas en función de la dialéctica social (poiesis) y moral (aisthesis) (Mélich, 1994: 168).

A lo anterior, se puede agregar que el carácter educativo participatorio constituye el núcleo del encuentro, por lo que "el hombre sólo puede participar en las realidades que ofrecen posibilidades a su acción creadora y reciben en alguna medida las posibilidades que él, a su vez otorga. Este doble ofrecimiento y aceptación de posibilidades contiene el entreveramiento de ámbitos" (Nussbaum, 1997: 150). Es, por consiguiente, en este encuentro de ámbitos que la participación se potencia como característica de una antropología pedagógica de la sensibilidad literaria.

Comprender el mundo de la novela a través de la imaginación narrativa prepara al lector para comprender la vida de otras personas, otros mundos, otros relatos y fortalece la idea de que el juegoy la creación son parte de la formación de la persona y del ciudadano, papel que desempeñan las instituciones escolares, por lo cual

para desempeñar bien su función en este sentido, las instituciones educativas deben adjudicar un rol protagónico a las artes y las humanidades $\mathrm{n}$ el programa curricular, cultivando un tipo de formación participativa que active y mejore la capacidad de ver el mundo a través de los ojos de otros ser humano (Nussbaum, 2010: 132).

Esta capacidad de ver el mundo a través de los ojos de los personajes de ficción, es lo que permite la comprensión y el reconocimiento a través de la imaginación

huelga decir que cuando la imaginación entra en juego, algunos lectores son bastante perezosos, mientras otros actúan con mayor presteza. Un escritor que satisface las imaginaciones más perezosas transmitirá de forma más explícita los sentimientos y pensamientos que los lectores debería sentir cuando una imagen concreta aparece en su mente. Mientras que un novelista que confía en el poder de la imaginación del lector se limitará a describir y definir con palabras las imágenes que constituyen los momentos de las novelas, y dejará los sentimientos y pensamientos en manos del lector para que sea este quien los defina (Pamuk, 2012). 
Pamuk concibe dos modos de escribir, a la vez que esboza las características del lector apropiado para cada tipo de novela, aunque sin perder de vista la imaginación. En este punto, la imaginación del lector, sea perezoso o sea activo, entra en juego creativo a partir del encuentro con la trama narrativa. Por lo tanto, El encuentro de ámbitos de lectura en el terreno de la trama narrativa, es un encuentro creativo mediado por la imaginación con la participación del lector y el antecedente inmediato de lo escrito en la obra narrativa. En este sentido, la imaginación narrativa contiene y supone el encuentro entre el ámbito de la trama narrativa desarrollada por el autor y el ámbito de mundo del lector, evidente en el acercamiento al texto y la comprensión de su sentido o sentidos.

En definitiva, la comprensión de obras literarias narrativas desde la perspectiva de la imaginación narrativa tiene como antecedente inmediato el reconocimiento como valor de la experiencia literaria, con todo lo que implica lograr el encuentro entre el mundo, el mundo de las novelas y el mundo del lector

Esta aproximación a la lectura con un enfoque cívico y evaluativo es moral y política. Plantea interrogantes sobre el modo en que la interacción del lector construye una amistad o comunidad, y nos invita a analizar los textos mediante evaluaciones morales y sociales de los tipos de comunidades que ellos crean (Nussbaum, 2005: 136).

En esta cita cabe la posibilidad de entender cómo concibe Nussbaum la lectura: con criterios morales y evaluativos en función de la comprensión del otro. En este punto es que surge el rechazo a esta manera de leer las novelas: para Nussbaum las novelas son fuente ejemplificante de análisis moral. En perspectiva de la lectura literaria, la comprensión lectora de obras literarias narrativas no debe ser concebida como una mediación ejemplificante en el análisis filosófico moral, ya que las obras literarias tienen su propia autonomía analítica: las obras mismas exigen su modo de leer el mundo fáctico, el mundo del texto y el mundo del lector.

\section{Conclusiones}

Para Martha Nussbaum la imaginación narrativa pone en juego la relación entre ficción y teoría moral (Kalin, 1992; Llanera, 2014; Hämäläinen, 2016). Con este supuesto, Hämäläinen (2016) esboza una comparación entre la teoría moral de Martha Nussbaum e Iris Murdoch, dos autoras que conjugan en sus análisis de lo literario y lo filosófico. En Nussbaum se rastrea y 
pone en evidencia el fundamento de la ética aristotélica y su visión de la razón práctica en la lectura de novelas; en Murdoch la visión platónica de la vida como un ideal. Llanera (2014), siguiendo esta ruta, indica que Martha Nussbaum es de estirpe aristotélica, al poner en consideración la cuestión moral al leer novelas y la desaparición de las fronteras entre el mundo individual y el mundo de los otros en la narración. El mundo de los otros en la ficción narrativa es uno de los rasgos de la imaginación narrativa (Andrews, 2014) y está asociado al autoexamen para la deliberación pública.

Desde la obra crítica sobre la imaginación literaria, se puede extraer que la revisión crítica se centra en el intento de Nussbaum para abolir las fronteras entre filosofía y literatura; además, se puntualiza que la imaginación en Nussbaum tiende a lo moral y a la justicia social, con un modo de leer la novela con fines de análisis moral. Los críticos ponen en duda la relación entre el valor literario de una obra y su valor moral, por cuanto la lectura de una novela es en sí misma una experiencia moral que no necesita ser puesta en evidencia en la relación literaria De otra parte, la categoría de imaginación narrativa tiene posibilidades educativas en lo que se refiere a educación literaria y educación emocional (Jiménez Bósquez, 2016) para la formación de ciudadanía, lo cual complementa la idea de comprensión de lo ajeno, de otras culturas, de otras personas. La lectura literaria permite una aproximación a esas culturas y personas y en concordancia con este modo de lectura puede surgir un tipo de la educación literaria que posibilita la lectura de obras literarias narrativas, lo cual garantizaría un encuentro de ámbitos de lectura de novelas: este encuentro se lleva a cabo entre el ámbito de la ficción narrativa y la experiencia literaria del lector. De esta forma, el lector de obras literarias narrativas se ve involucrado en una experiencia de imaginación literaria que lo lleva por el camino de una pedagogía de la experiencia literaria, capaz de fomentar respuestas de lectura empática hacia otros mundos, otras personas, otras vidas y, a la vez, desarrolla las posibilidades humanas de la imaginación narrativa como valor creativo, estético y comunicativo en la formación humanística.

\section{Bibliografía}

Andrews, M. (2014). Narrative imagination and everyday life. Oxford: Oxford University Press.

Antaki, M. (2012). The Turn to imagination in legal Theory: the re-enchantment of the world? Law Critique, 23, 1-20.

Bermúdez Pérez, E. P. (2014). La Literatura en la formación de ciudadanos. El desafío de una educación para la democracia. Revista Amauta, 23, 119-130. 
Bevan, R. (2006). Creed between the lines: the values and potential of literature in education. (Tesis de Maestría). Concordia University, Canadá.

Booth, W. (1998). Why Banning Ethical Criticism is a Serious Mistake. Philosophy and Literature, 22(2), pp. 366-393.

Buell, L. (1999). Introduction: in pursuits of ethics. PMLA (special topic: Ethics and Literary Studies), 114(1), 7-19.

Carroll, N. (2000). Art and ethical criticism: and overview of recent direction of research. Ethic, 110(2), 350-387.

Clavel, S. (2012). Martha Nussbaum et les usages de la Littérature en Philosophie Morale. Revue Philosophique, 1(137), 89-100.

Coeckelbergh, M. (2007). Imagination and principles. An Essay on the rol of imagination $n$ moral deliberation. New York: Palgrave-Macmillan.

Diamond, C. (1999). Martha Nussbaum and the need for the novels. En Adamson, J.; Freedman, R. y Parker, D. Renegotiating ethic in literature, philosophy and theory (pp. 39-64). Cambridge: Cambridge University Press.

Diamond, C. (1983). Having story about what Moral Philosophy is. New Literary History, 15(1), 155-169.

Fonnegra, C. P. (2012). La Narrativa como vía para la reflexión ética y política. Una aproximación a partir de los planteamientos teóricos de Paul Ricoeur, Hannah Arendt y Martha Nussbaum. (Tesis de Maestría). Universidad EAFIT, Colombia.

Hamilton, S. (2004). Ethics and Literature: love and perception in Henry James. (Tesis de Maestría). Louisiana State University, Estados Unidos.

Hämäläinen, N. (2016). Literature and moral theory. London: Bloombury.

Hänes, K. y Jansen, L. (2001). Reading for the good life? En Kallhoff, A. (Ed.). Martha C. Nussbaum: ethics and political philosophy (pp. 119-128). Müster: Lit Verlag.

Hidalgo, D. C. (2010). La Imaginación literaria como proyección del otro. Una reflexión desde la justicia poética de Martha Nussbaum. (Trabajo de grado). Universidad Tecnológica de Pereira, Colombia.

Horton, J. (2003). Life, literature and ethical theory: Martha Nussbaum on the role of literary imagination in ethical thought. En Horton, J. and Baumeister, A. Literature and political imagination (pp. 70-97). London: Routledge.

Jiménez Bósquez, D. A. (2016). Educación emocional para una ciudadanía cosmopolita. La propuesta de Nussbaum. Quito: Publicaciones de la Pontifica Universidad Católica del Ecuador. 
Johnson, P. (2004). Moral philosopher and the novel: a study Winch, Nussbaum and Rorty. London: Palgrave.

Kalin, J. (1992). Knowing novels: Nussbaum on fiction and moral theory. Ethics, 103, 135-151.

Koopman, E. M. y Hakemulder, F. (2015). Effects of literature on empathy and self-reflection: a theoretical-empirical framework. Journal of Literature Journal, 9(19), 79-111.

Llanera, T. (2014). Morality by words: Murdoch, Nussbaum, Rorty. Budhi: A Journal of Ideas and Culture, 18(1), 1-17.

Lourens, H. (2009). From Fiction to phronésis. A Critical dialogue with Martha Nussbaum on the importance of concrete fictional literature in moral philosophy. (Tesis de Maestría). Stellenbosch University, Sudáfrica.

Maxwell, B. (2006). Naturalized compassion: a critique of Nussbaum on literature as education form compassionate citizenry. Journal of Moral Education, 35(3), 335-352.

Mcgregor, R. F. (2013). The Autonomy of Literature. (Tesis Doctoral). University of York, Reino Unido.

Mélich, J. C. (1994). Del Extraño al cómplice. La educación en la vida cotidiana. Barcelona: Anthropos.

Nussbaum, M. C. (2010). Sin fines de lucro. Por qué la democracia necesita humanidades. Buenos Aires: Katz editors.

Nussbaum, M. C. (1998). Exactly and responsibly: a defense of ethical criticism. Philosophy and Literature, 22(2), 334-365.

Nussbaum, M. C. (2005). Cultivando la humanidad. Una defensa clásica de la reforma de la educación liberal (traducción J. Pailaya). Barcelona: Paidós.

Nussbaum, M. C. (1997). Justicia poética. La Imaginación literaria y la vida pública (traducción C. Gardini). Santiago de Chile: Editorial Andrés Bello.

Pamuk, O. (2012). Museos y novela. En El novelista ingenuo y el novelista sentimental (pp. 97119). Barcelona: Mondadori.

Posner, R. A. (1998). Against ethical criticism: part two. Philosophy and Literature, 22(2), 394412.

Posner, R. A. (1997). Against ethical criticism. Philosophy and Literature, 22(1), 1-27.

Putnam, H. (1983). Taking rules seriously: a response to Martha Nussbaum. New Literary History, 15(1), 193-200.

Reyes, Y. (2007). La casa imaginaria. Lectura y Literatura en la primera infancia. Bogotá: Norma. 
Robbins, W. E. (2015). Science Fiction is good for you too: a reply to Martha Nussbaum's theory of literary engagement. (A Thesis in partial fulfillments of the requirements for the degree Master of Arts). University of Saskatchewan, Canadá.

Singleton, J. (2006). Henry James- Aristotle's ally, an exclusive pact? Philosophy and Literature, 30(1), 61-78.

Stow, S. (2000). Unbecoming virulence: the political of the Ethical Criticism debate. Philosophy and Literature, 24, 185-196.

Voice, P. (1994). Why Literature cannot be Moral Philosophy. Theory, 83/84, 123-134.

Von Wright, M. (2002). Narrative imagination and taking the perspectives of other. Studies in Philosophy and Education, 21(4), 407-416.

Wollheim, R. (1983). Flawed Crystals James's the Golden Bowl and the plausibility of Literature and Moral Philosophy. New Literary History, 15(1), 185-191. 